\title{
Treatment of Neurological Manifestations of Gluten Sensitivity and Coeliac Disease
}

\author{
Panagiotis Zis, PhD \\ Marios Hadjivassiliou, MD*
}

\author{
Address \\ *Academic Department of Neurosciences, Sheffield Teaching hospitals NHS Trust \\ and University of Sheffield, Royal Hallamshire Hospital, Glossop Road, Sheffield, \\ S10 2JF, UK \\ Email: m.hadjivassiliou@sheffield.ac.uk
}

Published online: 26 February 2019

(C) The Author(s) 2019

This article is part of the Topical Collection on Neurologic Manifestations of Systemic Disease

Keywords Gluten sensitivity $\cdot$ Coeliac disease $\cdot$ Ataxia $\cdot$ Neuropathy $\cdot$ Gluten-free diet $\cdot$ Neurological manifestations

\section{Abstract}

Purpose of review The aim of this paper was to overview the current literature in order to establish the available treatment options for the neurological manifestations of glutenrelated disorders (serologically confirmed gluten sensitivity and coeliac disease).

Recent findings A range of debilitating neurological manifestations is increasingly being recognized in patients with gluten sensitivity with and without enteropathy even in the absence of gastrointestinal symptoms. Ataxia is the commonest neurological manifestation, followed by peripheral neuropathy. Epilepsy, headache, encephalopathy, various movement disorders, cognitive impairment, and muscle disorders have also been linked to gluten sensitivity and coeliac disease and are discussed in this review.

Summary Strict gluten-free diet is an effective first-line treatment of the neurological manifestations of gluten-related disorders. Very few patients will require additional immunosuppressive treatment usually in the form of mycophenolate.

\section{Introduction}

Gluten-related disorders (GRDs) represent a spectrum of diverse clinical entities sharing a common trigger: ingestion of gluten. Although the gastrointestinal (GI) manifestations of gluten sensitivity are the most prevalent, a range of debilitating neurological manifestations of gluten sensitivity is increasingly seen in clinical practice even in the absence of GI symptoms.

Coeliac disease (CD) is the best recognized GRD, where a small bowel enteropathy occurs in genetically 
susceptible individuals after exposure to the protein gliadin (found in wheat, barley, and rye) [1]. Noncoeliac gluten sensitivity (NCGS) is a term used by gastroenterologists to describe patients with primarily GI symptoms related to the ingestion of gluten who do not have enteropathy but who symptomatically benefit from a gluten-free diet (GFD) [2]. However, from a neurologist's perspective, we consider as gluten sensitive
(GS) only those patients with positive serology in the form of anti-gliadin IgG and/or IgA (AGA) with or without positivity for transglutaminase (TG) or endomysial antibodies (EMA) and with no evidence of enteropathy which is diagnostic of CD [3].

This paper is an overview of the current literature, aiming to report the available treatment options for the neurological manifestations of GS and CD.

\section{Gluten ataxia}

Gluten ataxia (GA) is defined as a sporadic ataxia with positive AGA [4-6]. In a series of 1500 patients with progressive ataxia evaluated over a period of more than 20 years at a specialist Ataxia Centre (Sheffield, UK), GA had a prevalence of $20 \%$ amongst all ataxias and more than $40 \%$ amongst idiopathic sporadic ataxias [7].

GA usually presents with pure cerebellar ataxia, occasionally in combination with sensory ataxia [8] or, rarely, ataxia in combination with myoclonus [9-11]. GA is usually of insidious onset; however, it can also be rapidly progressive mimicking paraneoplastic cerebellar degeneration. Gaze-evoked nystagmus is common. All patients have gait ataxia and the majority have lower limb ataxia. Less than $10 \%$ of patients with GA will have any GI symptoms; however, about half of the patients will have CD on biopsy.

Patients with GA usually have evidence of cerebellar atrophy on MR imaging with particular predilection for the cerebellar vermis. MR spectroscopy of the vermis is abnormal in all patients with GA (low N-acetyl aspartate/creatine ratio). Even in patients with GA without cerebellar atrophy, MR spectroscopy is abnormal, suggesting that MR spectroscopy is a useful monitoring tool [12•].

Loss of Purkinje cells in the cerebellum, the end result of prolonged gluten exposure in patients with GA, is irreversible; therefore, prompt treatment is more likely to result in improvement or stabilization of the ataxia. The current recommendation is that patients presenting with idiopathic progressive cerebellar ataxia should be screened for sensitivity to gluten using anti-gliadin IgG and IgA, anti-EMA, anti-TG2, and anti-TG6 (if available) antibodies. Patients positive for any of these antibodies with no alternative cause for their ataxia should be offered a specialist dietician review and to be advised to embark on a strict GFD, with regular follow-up to ensure that the antibodies are eliminated, which usually takes 6 to 12 months.

The response to treatment with GFD depends on the duration of the ataxia prior to the diagnosis of sensitivity to gluten. In a systematic case-controlled study of 40 patients presenting with ataxia, with or without an enteropathy, it was shown that patients who adhere to a strict GFD (having serological evidence of elimination of the anti-gliadin antibodies being on the diet) improved significantly in their performance in ataxia test scores and in the subjective global clinical impression scale compared to the control group, which comprised of patients opting not to embark on a GFD [13]. The two groups did not differ regarding age, gender, and severity of ataxia and, interestingly, the 
improvement was apparent even after excluding patients with an enteropathy.

Apart from the clinical improvement that GFD has on patients with GA, in a large study of 117 patients, it has been demonstrated that a GFD has a positive effect in the MR spectroscopy parameters too [12•]. In particular, The Nacetylaspartate (NAA)/creatine $(\mathrm{Cr})$ area ratio from the cerebellar vermis was increased in 62 out of $63(98 \%)$ patients on strict GFD (with elimination of anti-gliadin antibodies), in 9 of 35 (26\%) patients on GFD but positive antibodies, and in only 1 of 19 (5\%) patients not on GFD. The demonstration of increased NAA/Cr ratio on repeat scanning following strict GFD strengthens previous findings of clinical improvement of the ataxia in patients with GA. The presence of enteropathy is not a prerequisite for such improvement; therefore, patients with positive serology and negative duodenal biopsy should still be treated with strict GFD.

In a small uncontrolled case series of 4 patients with GA, Bürk et al. reported the use of intravenous immunoglobulins (IVIG) as having a positive effect; however, there was no information about the diet and serological status in these patients [14].

GA with myoclonus of cortical origin can be seen in refractory $C D$, where a GFD might not be adequate on its own. In the largest series of myoclonic ataxia secondary to refractory $\mathrm{CD}$ [11], five patients were treated with mycophenolate and one in addition with rituximab and IVIG. While their ataxia and enteropathy improved, the myoclonus remained the most disabling feature of their illness.

\section{Gluten neuropathy}

Gluten neuropathy (GN) is defined as an otherwise idiopathic sporadic largefiber neuropathy with serological evidence of sensitivity to gluten [15]. The commonest types is symmetrical sensorimotor axonal length dependent peripheral neuropathy (about 75\% of cases), followed by sensory ganglionopathy, an asymmetric form of pure sensory neuropathy [16] where the pathology is within the dorsal root ganglia [17-19]. Other types of large fiber neuropathies that have been reported include asymmetrical sensorimotor neuropathy (mononeuritis multiplex) and very rarely pure motor neuropathy.

Involvement of small fibers (A $\delta$ and $\mathrm{C}$ fibers) leads to small fiber neuropathy (SFN), which is characteristically painful (patients report a burning sensation mainly at the soles or the fingertips) or autonomic neuropathy [20-22]. Though the exact prevalence of gluten-related SFN is yet to be confirmed, it is known that more than half of the patients with GN report pain [23•], suggesting that small fibers are often involved.

Gluten neuropathy is a slowly progressive condition. About $25 \%$ of the patients will have evidence of enteropathy on biopsy (CD) but the presence or absence of an enteropathy does not influence the positive effect of a strict gluten-free diet. In a systematic, controlled study of the effect of GFD in a large series of patients with gluten neuropathy (of the sensorimotor axonal type), a clear clinical and neurophysiological improvement was demonstrated after 
12 months in those patients adhering to a strict GFD with serological elimination of the AGA [17]. In particular, there was significant increase in the sural sensory nerve action potential, the pre-defined primary endpoint, as well as subjective improvement of the neuropathic symptoms. Subgroup analysis showed that the capacity for recovery is less when the neuropathy is severe. In a large series of patients with gluten sensitivity and sensory ganglionopathy, it has been shown that strict adherence to a GFD may result in stabilization of the neuropathy irrespective of the presence of enteropathy [19].

In the largest - to date-cohort of patients with GN, of both the axonal type and sensory ganglionopathy, it was shown that GFD is protective as it is associated with a significant reduction in peripheral neuropathic pain associated with GN [23•].

GN can cause a significant burden in the overall quality of life (QoL) of patients with GN [24]. In a case-controlled study, it was shown that, compared to controls, patients with GN have worse scores in the physical functioning, role limitations due to physical health, energy/fatigue, and general health subdomains of the SF-36 questionnaire [24]. Interestingly after having adjusted for age, gender and disease severity being on a strict GFD correlates with better SF-36 scores in the pain domain and in the overall health change domain, suggesting that along with the clinical and the neurophysiological improvement, a strict GFD can lead to a better overall QoL [24].

\section{Other treatment options}

A beneficial effect of IVIG as an adjuvant option to GFD for the treatment of neuropathic pain associated to gluten-related SFN (2 patients) and GN (1 patient) with concomitant GA has been suggested by Souayah et al. [25]. Similarly, Anandacoomaraswamy et al. reported clinical improvement following IVIG and GFD implementation in an isolated case of a patient with a combination of GN and GA [26]. However, no reports or controlled trials of the use of IVIG in GN as a monotherapy are available, and therefore, the therapeutic potential of IVIG in GN remains questionable.

\section{Epilepsy due to gluten sensitivity}

Epileptic seizures presenting in the context of gluten sensitivity encompass the full spectrum of epilepsy and include both patients with and without overt brain pathology who may or may not respond to anti-epileptic drugs (AED). This spectrum includes a range of interesting pathological features including epilepsy and cerebral calcifications (CEC), hippocampal sclerosis, and temporal lobe epilepsy (TLE) in the context of gluten sensitivity and those who apparently display no pathological clues to the specific cause of the epilepsy [27•].

More than half of patients with epilepsy and GS or CD will respond positively to a GFD (defined as either decreased frequency of seizures with GFD, cessation of seizures with GFD, successful reduction of AED with initiation of GFD, or cessation of AED following the introduction of GFD). The response to GFD could reflect resolution/reduction of a neurological insult caused by gluten ingestion or be the result of improved absorption of AED due to resolution of gastrointestinal disturbance [28]. 
When considering specifically patients with CEC, a syndrome which refers patients with focal, medically refractory epilepsy and show parieto-occipitally brain calcifications on CT or MRI, response to AED alone appears to be poor with the majority (73\%) being unresponsive to treatment. Response to GFD appears to be more effective, with 53\% of patients managed with GFD demonstrating a good response. Interestingly, three prospective cohort studies appear to have demonstrated an inverse relationship between effectiveness of GFD and duration of epilepsy prior to GFD, perhaps due to increasing neurological damage [29-31].

\section{Gluten-related movement disorders}

\section{Headache and GS/CD}

In an extensive, recent, systematic review, Vinagre-Aragón et al. summarized all available literature regarding movement disorders (MD) other than GA related to GS or CD [32•].

At the moment, restless leg syndrome is the second commonest MD (GA is the commonest) with more than $50 \%$ of patients responding positively when embarking on a GFD, even as a monotherapy. Reports of patients with myoclonus follow, however, myoclonus appears to be treatment-resistant on both aggressive immunosuppression and GFD.

Less frequently reported MD include chorea, palatal tremor, dystonia, parkinsonism, opsoclonus-myoclonus, propriospinal myoclonus, paroxysmal dyskinesia, myorhythmia, and myokymia. No robust information about the effectiveness of a GFD exist in these MDs to date.

\section{Cognition and GS/CD}

Chronic migraines are affecting adult and children patients with CD and GS approximately two times more frequent compared to controls [33-35]. Female sex, depression, and anxiety are independent predictors of migraines, whereas age $>65$ years is protective [34]. Although there was no correlation between years on gluten-free diet and migraine severity [34], Lionetti et al. showed that a GFD has a beneficial effect (reduction of headache frequency in the majority of patients, with approximately $25 \%$ managing to become headache-free [33].

A possible association between progressive cognitive impairment and CD has been suggested in small case series $[36,37]$ and analysis of Swedish epidemiological data showed that although patients with CD are not at increased risk for dementia overall, subgroup analysis revealed that they may be at increased risk of vascular dementia [38].

Therapeutic options for patients with advanced dementia are not available, and even a GFD has no beneficial effect. However, in newly diagnosed CD, cognitive performance improves with adherence to a GFD in parallel to mucosal healing [39]. Therefore, a GFD should be introduced as soon as possible in patients with GS or CD as, even in the absence of a mild cognitive impairment, it has a potentially protective effect [40]. 
Table 1. Neurological manifestation of gluten-related disorders and their response to gluten free diet

\begin{tabular}{|c|c|c|c|}
\hline Gluten-related disorder & Type & Aspect & $\begin{array}{l}\text { Response to } \\
\text { gluten-free diet }\end{array}$ \\
\hline \multirow[t]{2}{*}{ Ataxia } & & Balance & Improvement \\
\hline & & $\begin{array}{l}\text { Magnetic resonance spectroscopy } \\
\text { parameters }\end{array}$ & Improvement \\
\hline \multirow[t]{4}{*}{ Peripheral neuropathy } & Sensory ganglionopathy & Neurophysiology & Stabilization \\
\hline & & Pain (small fiber neuropathy) & Improvement \\
\hline & Sensorimotor axonal & Neurophysiology & Improvement \\
\hline & & Pain (small fiber neuropathy) & Improvement \\
\hline \multirow[t]{3}{*}{ Epilepsy } & Idiopathic & Seizure frequency & Improvement \\
\hline & & Anti-epileptic drug reduction & Improvement \\
\hline & $\begin{array}{l}\text { Epilepsy and cerebral } \\
\text { calcification syndrome }\end{array}$ & Seizure frequency & Improvement \\
\hline \multirow[t]{2}{*}{ Movement disorders } & Restless leg syndrome & Symptoms & Improvement \\
\hline & Myoclonus & Symptoms & No \\
\hline Headache & Migraine & Frequency & $\begin{array}{l}\text { Improvement in } \\
\text { frequency, duration, } \\
\text { and intensity of } \\
\text { migraine }\end{array}$ \\
\hline \multirow[t]{2}{*}{ Cognition } & Advanced dementia & Cognition & No \\
\hline & Mild cognitive impairment & Cognition & Improvement \\
\hline \multirow[t]{2}{*}{ Myopathy } & & Symptoms & Improvement \\
\hline & & Creatine kinase levels & Improvement \\
\hline
\end{tabular}

\section{Gluten encephalopathy}

Although these neurological manifestations can present independently in patients with GS or CD, the term gluten encephalopathy was used to describe a combination of frequent, often intractable headaches, cognitive complaints (sometimes patients describe these as "foggy brain") and excessive, for age, white matter abnormalities on brain MR imaging [41]. Despite the fact that the white matter abnormalities do not resolve following a GFD, such diet arrests progression of the MRI changes and both the associated cognitive difficulties and headaches resolve.

\section{Gluten myopathy}

Proximal or isolated ocular myopathy cases in both adults and children have been described as isolated case reports over the last four decades 
$[42,43]$. In the majority, if not all the cases, the myopathy was the first manifestation of CD and GFD was proven to be effective in resolving the patients' symptoms. It is speculated that such acquired myopathies are related to deficiency in fat-soluble vitamin D or E, as a result of malabsorption secondary to CD which is reversed following a GFD. However, there have also been cases of inflammatory myopathy (myositis with or without inclusion bodies) associated with CD and, therefore, an immune-mediated mechanism to account for the myopathy has also been suggested [44].

Hadjivassiliou et al. presented the largest to date series of patients who presented with a clinical picture of a myopathy and in whom diagnostic work-up led to the diagnosis of gluten sensitivity [45]. The mean age at onset of the myopathic symptoms was 54 years. Inflammatory myopathy was the most common finding on neuropathological examination. Not all patients had enteropathy, suggesting that GS can also be linked to myopathy. Although some patients were on immunosuppressive treatment (i.e., azathioprine, methotrexate, prednisolone), some showed clinical improvement of the myopathy just with GFD. The improvement was also associated with reduction or normalization of serum creatine kinase level.

Table 1 summarizes the neurological manifestation of GRDs and their response to GFD.

\section{Conclusions}

Gluten-related disorders refer to a group of immune-mediated diseases triggered by ingestion of gluten proteins. While coeliac disease has been the most recognized entity within GRD, there is now clear evidence of extraintestinal manifestations of which those affecting the nervous system are proving to be very common. The presence of gastrointestinal symptoms, may offer a major potential advantage to those patients with gluten sensitivity, as it substantially increases their chances of being diagnosed and treated early, whereas the diagnosis of those patients presenting purely with extraintestinal manifestations may be delayed.

Treatment should include symptomatic management; however, the hallmark in gluten-related neurological manifestations is embarking on a strict GFD as soon as possible. In the majority of such diseases, with the exemption of cortical myoclonus and advanced dementia, GFD has a positive therapeutic effect. Immunosuppression is only used in cases where strict GFD alone has not been beneficial and in those patients with refractory $\mathrm{CD}$.

\section{Acknowledgments}

This is a summary of independent research supported by BRC and carried out at the National Institute for Health Research (NIHR) Sheffield Clinical Research Facility. The views expressed are those of the authors and not necessarily those of the BRC, NHS, the NIHR, or the Department of Health. 


\section{Compliance with Ethical Standards}

\section{Conflict of Interest}

The authors declare that they have no conflicts of interest.

Human and Animal Rights and Informed Consent

This article does not contain any studies with human or animal subjects performed by any of the authors.

\section{Open Access}

This article is distributed under the terms of the Creative Commons Attribution 4.0 International License (http://creativecommons.org/licenses/by/4.0/), which permits unrestricted use, distribution, and reproduction in any medium, provided you give appropriate credit to the original author(s) and the source, provide a link to the Creative Commons license, and indicate if changes were made.

\section{Publisher's Note}

Springer Nature remains neutral with regard to jurisdictional claims in published maps and institutional affiliations.

\section{References and Recommended Reading}

Papers of particular interest, published recently, have been highlighted as:

- Of importance

1. Ludvigsson JF, Bai JC, Biagi F, Card TR, Ciacci C, Ciclitira PJ, et al. Diagnosis and management of adult coeliac disease: guidelines from the British society of gastroenterology. Gut. 2014;63(8):1210-28.

2. Sapone A, Bai JC, Ciacci C, Dolinsek J, Green PH, Hadjivassiliou $\mathrm{M}$, et al. Spectrum of gluten-related disorders: consensus on new nomenclature and classification. BMC Med. 2012;10:13. https://doi.org/10. 1186/1741-7015-10-13.

3. Hadjivassiliou M, Gibson A, Davies-Jones GAB, Lobo AJ, Stephenson TJ, Milford-Ward A. Does cryptic gluten sensitivity play a part in neurological illness? Lancet. 1996;347:369-71.

4. Hadjivassiliou M, Grünewald RA, Chattopadhyay AK, Davies-Jones GA, Gibson A, Jarratt JA, et al. Clinical, radiological, neurophysiological and neuropathological characteristics of gluten ataxia. Lancet. 1998;352:1582-5.

5. Hadjivassiliou M, Grünewald R, Sharrack B, Sanders D, Lobo A, Williamson C, et al. Gluten ataxia in perspective: epidemiology, genetic susceptibility and clinical characteristics. Brain. 2003;126:685-91.
6. Hadjivassiliou M, Aeschlimann P, Sanders DS, Mäki M, Kaukinen K, Grünewald RA, et al. Transglutaminase 6 antibodies in the diagnosis of gluten ataxia. Neurology. 2013;80(19):1740-5.

7. Hadjivassiliou M, Martindale J, Shanmugarajah P, Grünewald RA, Sarrigiannis PG, Beauchamp N, et al. Causes of progressive cerebellar ataxia: prospective evaluation of 1500 patients. Neurol Neurosurg Psychiatry. 2017;88(4):301-9.

8. Zis P, Sarrigiannis PG, Rao DG, Hoggard N, Sanders DS, Hadjivassiliou M. Cerebellar ataxia with sensory ganglionopathy; does autoimmunity have a role to play? Cerebellum Ataxias. 2017;4:20.

9. Lu CS, Thompson PD, Quin NP, parkes JD, Marsden CD. Ramsay Hunt syndrome and coeliac disease: a new association. Mov Disord. 1986;1:209-19.

10. Bhatia KP, Brown P, Gregory R, Lennox GG, Manji H, Thompson PD, et al. Progressive myoclonic ataxia associated with coeliac disease. The myoclonus is of cortical origin, but the pathology is in the cerebellum. Brain. 1995; 118(Pt 5):1087-93.

11. Sarrigiannis PG, Hoggard N, Aeschlimann D, Sanders DS, Grünewald RA, Unwin ZC, et al. Myoclonus ataxia 
and refractory coeliac disease. Cerebellum Ataxias. 2014;1(1):11. https://doi.org/10.1186/2053-8871-111.

12. Hadjivassiliou M, Grunewald RA, Sanders DS, Shanmugarajah P, Hoggard N. Effect of gluten-free diet on MR spectroscopy in gluten ataxia. Neurology. 2017;89:1-5.

Demonstrates the effectiveness of gluten-free diet on cerebellar spectroscopy.

13. Hadjivassiliou M, Davies-Jones GAB, Sanders DS, Grünewald RAG. Dietary treatment of gluten ataxia. J Neurol Neurosurg Psychiatry. 2003;74(9):1221-4.

14. Bürk K, Melms A, Schulz JB, Dichgans J. Effectiveness of intravenous immunoglobulin therapy in cerebellar ataxia associated with gluten sensitivity. Ann Neurol. 2001;50:827-8.

15. Zis $P$, Rao DG, Sarrigiannis $P G$, Aeschlimann $P$, Aeschlimann DP, Sanders D, et al. Transglutaminase 6 antibodies in gluten neuropathy. Dig Liver Dis. 2017;49(11):1196-200.

16. Zis $\mathrm{P}$, Hadjivassiliou M, Sarrigiannis PG, Barker ASJE, Rao DG. Rapid neurophysiological screening for sensory ganglionopathy: a novel approach. Brain Behav. 2017;7(12):e00880. https://doi.org/10.1002/brb3. 880 .

17. Hadjivassiliou M, Kandler RH, Chattopadhyay AK, Davies-Jones AG, Jarratt JA, Sanders DS, et al. Dietary treatment of gluten neuropathy. Muscle Nerve. 2006;34(6):762-6.

18. Hadjivassiliou $M$, Rao DG, Grìnewald RA, Aeschlimann DP, Sarrigiannis PG, Hoggard N, et al. Neurological dysfunction in coeliac disease and noncoeliac gluten sensitivity. Am J Gastroenterol. 2016;111(4):561-7.

19. Hadjivassiliou M, Rao DS, Wharton SB, Sanders DS, Grunewald RA, Davies-Jones GAB. Sensory ganglionopathy due to gluten sensitivity. Neurology. 2010;75:1003-8.

20. Hadjivassiliou M, Chattopadhyay AK, Davies-Jones GAB, Gibson A, Grunewald RA, Lobo AJ. Neuromuscular disorder as a presenting feature of celiac disease. J Neurol Neurosurg Psychiatry.

1997;63:770-5.

21. Brannagan TH, Hays AP, Chin SS, Sander HW, Chin RL, Magda P, et al. Small-fiber neuropathy/ neuronopathy associated with celiac disease: skin biopsy findings. Arch Neurol. 2005;62:1574-8.

22. Gibbons CH, Freeman R. Autonomic neuropathy and celiac disease. J Neurol Neurosurg Psychiatry. 2005;76:579-81.

23. Zis P, Sarrigiannis PG, Rao DG, Hadjivassiliou M. Gluten neuropathy: prevalence of neuropathic pain and the role of gluten-free diet. J Neurol. 2018;265:2231-6. https://doi.org/10.1007/s00415018-8978-5.

Demonstrates the effectiveness of gluten-free diet in peripheral neuropathic pain.

24. Zis P, Sarrigiannis PG, Rao DG, Hadjivassiliou M. Quality of life in patients with gluten neuropathy: a case-controlled study. Nutrients 2018 May 23;10(6). pii: E662. doi: https://doi.org/10.3390/nu10060662.

25. Souayah N, Chin RL, Brannagan TH, Latov N, Green $\mathrm{PH}$, Kokoszka A, et al. Effect of intravenous immunoglobulin on cerebellar ataxia and neuropathic pain associated with celiac disease. Eur J Neurol. 2008 Dec;15(12):1300-3.

26. Anandacoomaraswamy D, Ullal J, Vinik AI. A 70-yearold male with peripheral neuropathy, ataxia and antigliadin antibodies shows improvement in neuropathy, but not ataxia, after intravenous immunoglobulin and gluten-free diet. J Multidiscip Healthc. 2008 Oct 1;1:93-6.

27. Julian T, Hadjivassiliou M, Zis P. Gluten sensitivity and epilepsy: a systematic review. J Neurol. 2018. https:// doi.org/10.1007/s00415-018-9025-2.

Systematically reviews epilepsy related to gluten sensitivity and provides information about the effectiveness of gluten-free diet.

28. Cronin CC, Jackson LM, Feighery C, Shanahan F, Abuzakouk M, Ryder DQ, et al. Coeliac disease and epilepsy. QJM. 1998;91(4):303-8.

29. Licchetta L, Bisulli F, Di Vito L, La Morgia C, Naldi I, Volta U, et al. Epilepsy in coeliac disease: not just a matter of calcifications. Neurol Sci. 2011;32(6):106974.

30. Arroyo HA, De Rosa S, Ruggieri V, de Dávila MT, Fejerman N. Argentinean Epilepsy and Celiac Disease Group. Epilepsy, occipital calcifications, and oligosymptomatic celiac disease in childhood. J Child Neurol. 2002;17(11):800-6.

31. Gobbi G, Bouquet F, Greco L, Lambertini A, Tassinari CA, Ventura A, et al. Coeliac disease, epilepsy, and cerebral calcifications. The Italian Working Group on Coeliac Disease and Epilepsy. Lancet. 1992;340(8817):439-43.

32. Vinagre-Aragón A, Zis P, Grunewald RA, Hadjivassiliou $M$. Movement disorders related to gluten sensitivity: a systematic review. Nutrients. 2018;10(8):E1034. https://doi.org/10.3390/nu10081034.

Systematically reviews movement disorders related to gluten sensitivity and provides information about the effectiveness of gluten-free diet.

33. Lionetti E, Francavilla R, Maiuri L, Ruggieri M, Spina M, Pavone $\mathrm{P}$, et al. Headache in pediatric patients with celiac disease and its prevalence as a diagnostic clue. J Pediatr Gastroenterol Nutr. 2009 Aug;49(2):202-7.

34. Dimitrova AK, Ungaro RC, Lebwohl B, Lewis SK, Tennyson CA, Green MW, et al. Prevalence of migraine in patients with celiac disease and inflammatory bowel disease. Headache. 2013;53(2):344-55.

35. Nenna R, Petrarca L, Verdecchia P, Florio M, Pietropaoli N, Mastrogiorgio G, et al. Celiac disease in a large cohort of children and adolescents with recurrent headache: a retrospective study. Dig Liver Dis. 2016;48(5):495-8.

36. Collin P, Pirttilä T, Nurmikko T, Somer H, Erilä T, Keyriläinen $\mathrm{O}$. Celiac disease, brain atrophy, and dementia. Neurology. 1991;41(3):372-5. 
37. Hu WT, Murray JA, Greenaway MC, Parisi JE, Josephs KA. Cognitive impairment and celiac disease. Arch Neurol. 2006;63(10):1440-6.

38. Lebwohl B, Luchsinger JA, Freedberg DE, Green PH, Ludvigsson JF. Risk of dementia in patients with celiac disease: a population-based cohort study. J Alzheimers Dis. 2016;49(1):179-85.

39. Lichtwark IT, Newnham ED, Robinson SR, Shepherd SJ, Hosking P, Gibson PR, et al. Cognitive impairment in coeliac disease improves on a gluten-free diet and correlates with histological and serological indices of disease severity. Aliment Pharmacol Ther. 2014;40(2):160-70.

40. Makhlouf S, Messelmani M, Zaouali J, Mrissa R. Cognitive impairment in celiac disease and non-celiac gluten sensitivity: review of literature on the main cognitive impairments, the imaging and the effect of gluten free diet. Acta Neurol Belg. 2018;118(1):21-7.
41. Hadjivassiliou M, Grünewald RA, Lawden M, DaviesJones GA, Powell T, Smith CM. Headache and CNS white matter abnormalities associated with gluten sensitivity. Neurology. 2001;56:385-8.

42. Hardoff D, Sharf B, Berger A. Myopathy as a presentation of coeliac disease. Dev Med Child Neurol. 1980;22(6):781-3.

43. Sandyk R, Brennan MJ. Isolated ocular myopathy and celiac disease in childhood. Neurology. 1983;33(6):792.

44. Kleopa KA, Kyriacou K, Zamba-Papanicolaou E, Kyriakides T. Reversible inflammatory and vacuolar myopathy with vitamin $\mathrm{E}$ deficiency in celiac disease. Muscle Nerve. 2005;31(2):260-5.

45. Hadjivassiliou M, Chattopadhyay AK, Grünewald RA, Jarratt JA, Kandler RH, Rao DG, et al. Myopathy associated with gluten sensitivity. Muscle Nerve. 2007;35(4):443-50. 